\title{
Do Programme Coordinators Contribute to the Professional Development of Residents? An Exploratory Study
}

\author{
Mayumi Aono \\ Gifu University \\ Haruo Obara \\ Okinawa Chubu Hospital \\ Chihiro Kawakami \\ Gifu University \\ Rintaro Imafuku \\ Gifu University \\ Takuya Saiki \\ Gifu University \\ Michael A. Barone \\ Johns Hopkins University School of Medicine \\ Yasuyuki Suzuki ( $\nabla$ ysuz@gifu-u.ac.jp ) \\ Gifu University
}

\section{Research Article}

Keywords: programme coordinator, educational role, resident, professional identity formation

Posted Date: November 9th, 2021

DOI: https://doi.org/10.21203/rs.3.rs-1054815/v1

License: (9) This work is licensed under a Creative Commons Attribution 4.0 International License. Read Full License 


\section{Abstract}

Background: With the development of training programmes for health professions, the role of programme coordinators has become increasingly important. However, their role in providing educational support for the professional development of resident trainees has not been well investigated. This study aimed to qualitatively analyse the involvement of programme coordinators in educational support for residents.

Methods: Semi-structured reflective writing on "support for residents" was collected from programme coordinators in teaching hospitals in Japan in 2017-18 using a web-based questionnaire. Descriptions were qualitatively analysed thematically, using the professional identity formation (PIF) framework.

Results: A total of 39 cases of "support for residents" by 31 coordinators were analysed. We found that prior personal problems, including mental health issues and insufficient social skills/unprofessional behaviour, were the most common issues that residents faced. Thematic analysis revealed that coordinators played a variety of educational roles: 1) requesting supervisors to re-consider their teaching; 2) protecting residents from the negative influence of clinical experiences; 3 ) facilitating residents' selfassessment and confidence; 4) creating a safer learning environment; 5) providing support for prior personal problems, 5-1) fostering a better atmosphere for the mental health of residents, 5-2) intervening for residents with insufficient social skills/unprofessional behaviour; 6 ) providing support for isolated residents; and 7) preventing problems with peers.

Conclusions: This study identified seven educational roles of programme coordinators for residents from a standpoint of PIF of residents. It also discussed four valuable attributes for coordinators: nonhierarchical relationships with residents, parenting attitudes, sensitivity to residents' change, and the perspective of a member of the public. These attributes would underpin coordinators' educational roles and facilitate the professional development of residents. This study provides a basis for defining and revising the role profiles of programme coordinators.

\section{Background}

Training programmes for health professions are developed worldwide. Accordingly, defining the roles and functions of non-medical programme coordinators who administratively support and manage training programmes is becoming increasingly important. ${ }^{1}$ Programme coordinators have direct contact with trainees throughout the training period and, in addition to performing important administrative and managerial functions such as trainee assessment and programme accreditation, coordinators may also be expected to manage professional development problems that trainees face by supporting them personally.

Programme coordinators are now considered to be integral members of the residency leadership team, ${ }^{2}$ and Dubois et al. ${ }^{3}$ claimed that the title of "programme coordinator" no longer seems adequate to represent the position. Instead, proposed titles such as programme manager, administrator, or educational specialist seem to fit in better. The authors also pointed out that a higher level of coordinator 
competency would be necessary to improve the quality of the training programmes and provide support for residents who are experiencing training difficulties. Job descriptions of programme coordinators have been reported from various fields, including surgery, ${ }^{2}$ radiology, ${ }^{4}$ and orthopaedics, ${ }^{5}$ where their role has expanded far beyond administrative functions; however, the specifics of the educational support provided by programme coordinators for the professional development of residents have not been well investigated.

Hu et al. ${ }^{6}$ conducted a qualitative analysis of 22 female coordinators managing undergraduate clinical training and revealed that their roles were emotional, informal, and related to their personal orientation. Some coordinators were like nurturing parents and identified with the "mother hen" persona, while referring to the students as "chicks". ${ }^{6}$ The authors argued that this image came from societal expectations of women as caring beings responsible for the welfare and outcomes of the young. Nickels et al. ${ }^{7}$ reported that non-physician staff members, including 19 administrative staff, felt comfortable providing feedback on the professional behaviour of residents, suggesting an expansion of the coordinators' role. Our prior work demonstrated that coordinators in Japan recognized the importance of educational support roles, including the parental role, for trainees. ${ }^{8}$

Cruess et al. established a schematic representation of the professional identity formation (PIF) and socialization of medical students and residents. ${ }^{9,10}$ Role models/supervisors/mentors and clinical/nonclinical experiences play a crucial role in facilitating trainees' PIF and socialization into a community of health professionals; peers, other health professionals, patients, families, friends, and the public also influence trainees' PIF significantly. ${ }^{10}$ In this way, programme coordinators are likely to also play an important role in the professional development and PIF of trainees.

This study, therefore, aimed to analyse the educational roles of programme coordinators, by adopting a PIF model as a theoretical framework for qualitative analysis. Specifically, the following research questions "How are programme coordinators involved in the professional development of trainees?" and "How do they support the professional development of trainees?" were formulated.

\section{Methods}

\section{Setting}

This study analyses the interaction of residents with programme coordinators in Japan. Medical training in Japan includes a six-year undergraduate medical school programme, ${ }^{11}$ followed by a two-year mandatory junior residency programme introduced in $2004 .{ }^{12,13}$ Residents rotate through internal medicine, surgery, emergency medicine, paediatrics, obstetrics and gynaecology, psychiatry, community medicine, and electives. ${ }^{13}$ Junior residency programmes are accredited by the Ministry of Health, Labour, and Welfare of Japan, and each teaching hospital must deploy a certain number of qualified supervising 
doctors. The qualifications, competencies, and role profiles of programme coordinators in this context are not well-established and their roles may vary on a case-by-case basis.

\title{
Study design
}

We conducted a qualitative ${ }^{14,15}$ content analysis ${ }^{16}$ of the formal and informal educational support provided by programme coordinators to residents across Japan in 2017-18, employing socialization factors of PIF 10,17 as the theoretical framework. We used a web-based semi-structured questionnaire to collect coordinators' responses on their experiences in supporting residents (Table 1). The instrument was designed as case-based, wherein coordinators' described an example event in which they provided support; this included general advice, advice on professional life and career, supporting residents with mental health issues, negotiation with supervisors and hospital administration, improvement of the learning environment, and other support for residents.

Table 1

Case description in semi-structured web questionnaire

\begin{abstract}
Please describe impressive cases in which you gave advice/support for residents' personal/professional development. Not only include cases within your official responsibility but also cases outside your responsibility carried out as a senior or relative of the resident.
\end{abstract}

Case Years after graduation, gender

Problem What was the resident's challenge or problem? How did you notice or become aware of the issue?

Support What advice did you provide to the resident? How did you support him/her?

Results Describe any change in the behaviour, thoughts, the impression of the resident, and changes in the learning environment. Describe any changes made by coordinators, supervisors, programme directors, or the organization.

Comments Provide any additional comments you feel are relevant.

\section{Theoretical framework}

Socialization factors of PIF proposed by Cruess et al. ${ }^{10,17}$ were used as the theoretical framework. Cruess et al. established a schema of PIF according to which novice students/trainees develop their professional identity through various experiences, which are categorized into socialization factors. ${ }^{10}$ In this study, each case described by the coordinators was qualitatively analysed based on the socialization factors of PIF as follows: 1) role models/supervisors/mentors, 2) clinical/non-clinical experiences, 3) formal teaching/self-assessment, 4) learning environment, 5) prior personal problems, including mental issues and insufficient social skills/unprofessional behaviours, 6) isolation from family, friends, and peers, and 7) problems with patients and peers.

\section{Participants}

We sent letters of invitation for this research to the administrative offices of 315 teaching hospitals in Japan, including urban, rural, public, and private hospitals which are accredited by the Ministry of Health, 
Labour, and Welfare of Japan, and quality-assured by the Japan Council for Evaluation of Postgraduate Clinical Training. Sixty individual coordinators who participated in our previous study were also invited directly. ${ }^{8}$ Forty-three coordinators ( 28 female and 15 male) from 38 hospitals ( 12 hospitals in large cities and 26 in other regions) agreed to participate in this study. Nineteen percent of the participants had worked as coordinators for more than six years, $29 \%$ for three to six years, and $52 \%$ for less than three years.

\section{Data collection}

An anonymous, case-based semi-structured questionnaire was used to collect data from the participants via the web (Table 1). We developed the questionnaire to ensure confidentiality, and considering the potential sensitivity of reported cases, to minimise hesitation in reporting experiences. Thirty-one out of the 43 coordinators who agreed to participate in this study responded to the web-based questionnaire (response rate $72 \%$ ); they reported 44 resident cases.

\section{Data analysis}

The research team comprised seven researchers from different backgrounds: a coordinator (MA), a general practitioner ( $\mathrm{HO}$ ), a nurse educator (CK), an education researcher (RI) and medical educators (TS, $M A B, Y S)$. The researchers reviewed case descriptions multiple times to better interpret what the participants reported (familiarization with data phase ${ }^{14}$ ). Two researchers (MA and YS) were independently involved in the initial coding, then MA, YS, and MAB cross-checked their interpretation and analysis (coding phase/generating initial themes phase ${ }^{14}$ ). The themes and findings were reviewed, discussed, and refined by all members of the research team (reviewing themes phase/defining and naming themes phase ${ }^{14}$ ), and all researchers came to a consensus on all final themes.

\section{Results}

\section{Coordinator support}

A total of 44 cases of resident support by programme coordinators were collected. Five of them were omitted due to insufficient information and 39 cases were analysed. The supplementary table shows the categorization of socialization factors, issues of residents, and responses of coordinators. Content analysis ${ }^{16}$ revealed that prior personal problems, including mental issues and insufficient social skills/unprofessional behaviours, were most frequent ( 16 cases), followed by isolation from family/friends/peers (6 cases), problems with peers ( 6 cases), role models (supervisors)/mentors (4 cases), formal teaching/self-assessment (4 cases), learning environment ( 2 cases) and clinical experiences (1 case).

\section{Thematic analysis of support by coordinators}

Key roles of and educational support by coordinators emerged for the seven socialization factors of PIF 10 through the thematic analysis (Table 2). 
Table 2

Educational roles of programme coordinators

\begin{tabular}{|ll|l|l|l|l}
\hline Socialization factors of PIF & Roles of programme coordinators \\
models/supervisors/mentors & 1. Requesting supervisors to re-consider their teaching \\
$\begin{array}{l}\text { Clinical/non-clinical } \\
\text { experiences }\end{array}$ & $\begin{array}{l}\text { 2. Protecting residents from the negative influence of clinical } \\
\text { experiences }\end{array}$ \\
\hline $\begin{array}{l}\text { Formal teaching/self- } \\
\text { assessment }\end{array}$ & 3. Facilitating residents' self-assessment and confidence \\
\hline Learning environment & 4. Creating a safer learning environment \\
\hline Prior personal problems & $\begin{array}{l}\text { 5. Providing support for prior personal problems } \\
\text { 5-1. Fostering a better atmosphere for the mental health of residents } \\
\text { 5-2. Intervening for residents with insufficient social } \\
\text { skills/unprofessional behaviour }\end{array}$
\end{tabular}

Isolation from

family/friends/peers

6. Providing support for isolated residents

Problems with patients and peers

7. Preventing problems with peers

\section{Requesting supervisors to re-consider their teaching}

Role models/supervisors/mentors strongly influence residents' thinking and behaviour as professionals; however, some supervisors are overly strict or have opposing opinions and severe supervision attitudes. The strict attitude and opposing opinions of supervisors can confuse residents and create an awkward relationship between supervisors and residents, resulting in the inhibition of professional development. Coordinators in our study were sensitive to the residents' stressors and behaviours; noticing their mood changes and responding quickly to improve their relationships with supervisors. Coordinators provided an opportunity for communication between residents and supervisors, requested supervisors to reconsider their strict teaching styles and proposed a review of teaching manuals in practice.

"She was a tough resident physically and mentally and she seemed to communicate with her strict supervisor well, however, I (the coordinator) felt that she was under stress based on her facial expression. I talked to her and the supervisor and arranged the opportunity to for them to talk and reflect with each other. I also conveyed her a message to reassure that I will take care of her and she can say anything to me at any time." (Case 3)

\section{Protecting residents from the negative influence of clinical experiences}


Powerful clinical experiences, such as medical errors and patient deaths, have a strong influence on the professional development of residents, both positively and negatively. Coordinators protected and supported residents, particularly from negative clinical experiences, by closely watching them, listening to them, and guiding them when required. Reflection and review of daily teaching by supervisors were also facilitated by coordinators.

"The resident had a medical accident that resulted in the death of a patient, and he took a leave from work for a while. A hospital team including me (the coordinator) was organized for his care. I made a lot of efforts including listening to him and watching him. The relationship between the resident and the supervisor was reviewed, and the supervisor said that he did not care for the resident and left him alone in the daily busywork. The team members worked together to provide psychological support and to protect the resident. He completed the training as planned, and he is now very active." (Case 5)

\section{Facilitating residents' self-assessment and confidence}

Residents sometimes have low confidence regarding their clinical competence and future career. Coordinators noticed subtle changes in their behaviour and appearance and speculated decreased selfconfidence. Coordinators reassured the residents, made arrangements for skill training to facilitate and improve their confidence, and created an amiable atmosphere.

"The resident was not confident with her blood sampling technique, and she looked depressed a little. I (the coordinator) spoke to her, when she came into my office, that no resident can do blood sampling perfectly from the beginning. I lent her a practice kit for blood sampling and secured a space for her to practice freely. She seemed to be confident somehow, and she came and asked me to lend other practice kits without hesitation thereafter." (Case 7)

\section{Creating a safer learning environment}

A positive and safe training environment promotes professional development; however, high workload and stress, and hostile and exclusionary human relationships can adversely impact professional development. Coordinators proposed improvements for resident workload to medical directors, and acted as a bridge between the residents and medical team members. Coordinators also proposed a review of instructional guides in a resident-centred way for the improvement of the learning environment.

"The resident seemed overworked, and had trouble with his colleagues. I (the coordinator) noticed his dissatisfaction from his tone of voice and facial expression. I talked frequently to him, even the smallest things. To reduce his workload, I negotiated with the programme director that this resident be given a week's leave and mental health advice twice a year. I found that the training in our hospital was conducted effectively and the stress of residents seemed to decrease thereafter as it acted as a bridge between residents and supervisors." (Case 10)

\section{Providing support for prior personal problems}


Sixteen out of the 39 residents (41\%) had prior personal problems, such as mental health issues, insufficient social skills, and unprofessional behaviours. Coordinators provided support to these residents through various means.

\section{5-1. Fostering a better atmosphere for the mental health of residents}

Residents may struggle to adapt to the dynamic changes of roles and positions, especially during the transition from student life to resident life. Coordinators attempted to create a relaxed atmosphere for these residents and welcomed them to visit and interact with them. Coordinators took the role of the main point of contact for residents with mental health issues, on behalf of busy supervisors, including watching and listening to residents' problems in a non-hierarchical equal relationship.

"Shortly after joining the programme he (the resident) fell ill and complained that he was not improving. I (the coordinator) assumed that he might have some mental health problem and I tried to figure out the cause of his stress. Every morning, we took time and talked to each other. His mental problem was due to concerns about a family problem as well as the training. I referred him to a specialist counsellor; in addition, I shared his information with a small number of supervisors with his permission. He seemed to feel more comfortable talking to me. I thought we (coordinators) should be more careful during times of change in the resident's environment such as at the beginning of training." (Case 18)

\section{5-2. Intervening for residents with insufficient social skills/unprofessional behaviour}

Some residents suffered because of their insufficient social skills or unprofessional behaviours which were difficult to remediate. Many of the issues were related to multiple factors, including mental health problems and the training environment/programme. Coordinators provided remediation for these residents by arranging meetings with the medical team and communicating with affiliated organizations. Coordinators tried to maintain a non-hierarchical relationship, listened to the resident, and created a positive atmosphere; however, some of the cases in this category were challenging and difficult to solve.

"The resident was asked by his supervisor to attend a conference and take a role of respondent, however, on the day of the conference, the resident suddenly cancelled and the supervisor had to ask another resident to take over the role. The resident insisted that he did not accept the supervisor's proposal and never apologized. As the atmosphere between them was bad, I (the coordinator) arranged the opportunity to for them to talk to each other and asked them to share their feelings. The atmosphere of the meeting was mature and the relationship was improved superficially, although I could not know the actual feeling between them." (Case 26)

\section{Providing support for isolated residents}

Some degree of isolation from family and friends, and independence from peers, is expected during the professional development and identity formation of trainees. ${ }^{17}$ However, the distress of isolation paired 
with participation in an unfamiliar environment may hinder socialization. Residents who graduate from a different medical school and are isolated from previous classmates sometimes have difficulties in adapting to the training programme. Coordinators identified residents who appeared to be having problems due to being isolated from families, friends, and peers. Coordinators supported these residents by inviting them to their office, creating a relaxed atmosphere, talking to them with a non-hierarchical attitude, and reassuring them.

"Her (the resident's) mother was sick and died. After the funeral, she returned to work, but her grief could not be healed and she took an additional leave for three weeks. When she saw the patients brought in by ambulance, she remembered her mother and said it was hard for her. I (the coordinator) provided an environment where she could take a break, and the emergency duty was suspended for several months, after consulting with the director. As time passed, she had regained her brightness, and a year after completing the training, she told me that she got married." (Case 28)

\section{Preventing problems with peers}

Relationships with peers, health professionals, and patients also have a significant impact on the promotion or inhibition of the professional identity development of residents. ${ }^{17}$ In our study, problems with peers or health professionals were often identified, and coordinators made efforts to prevent such instances. Coordinators arranged for opportunities to foster communication between residents and peers, advised residents that peer evaluation was important, and facilitated the residents' self-reflection.

"The resident seemed to be gentle, but I (the coordinator) noticed a problem with him when I received multi-source feedback from other health professionals. There were harsh comments such as "he is too loud" and "he is arrogant". He denied these behaviours at the beginning. I tried to listen to his thoughts and reasons, and I explained the importance of evaluation by colleagues. We talked about it repeatedly. Afterwards, he was able to think about his colleagues and communicate well with the staff. The supervisors trusted him, and he became one of the best residents. I value the personality of each resident and try to be upfront with them so as not to hurt their pride. When the supervisors don't want to say something or don't want to get angry with the resident, I will go in the middle and convey the message from the supervisor to the resident. (Case 36)

Table 3. Suggested attributes for programme coordinators

1. Non-hierarchical relationships with residents

2. Parenting attitudes to residents: supporting, fostering, and protecting

3. Sensitive to early and subtle changes in residents

4. Perspective of a member of the public 


\section{Discussion}

This is the first study investigating the educational roles of programme coordinators for the benefit of residents; specifically, the first one linking these roles to critical aspects of PIF 10 in trainees, to the best of our knowledge. In our study, prior underlying personal problems including mental health issues and insufficient social skills/unprofessional behaviours, were the most common socialization factors supported by coordinators, followed by isolation from family/friends/peers, problems with peers, and behaviours of supervisors/mentors. The National Survey of Internal Medicine Residency Program Directors in the USA revealed that $6.9 \%$ of residents faced problems, including medical knowledge, clinical judgement, unsatisfactory behaviours, and $42 \%$ of these residents had an underlying cause of situational, personal, or professional stress. ${ }^{18}$ This research did not include programme coordinators as individuals who identified these problem residents. The present study suggests that coordinators are additional and valuable staff to identify the residents in trouble. Reamy et al. reported that 21 out of 230 family medicine residents faced problems, including attitudinal problems (9 cases), interpersonal conflicts (6 cases), psychiatric illnesses (5 cases), family stress (3 cases), and relationship disruption (2 cases). ${ }^{19}$ Many of these troubles could be re-categorized into socialization factors of PIF, as we suggested.

Seven educational roles including two sub-roles of coordinators for residents emerged through the thematic analysis. Our study provides evidence that coordinators can take important roles beyond the scope of administration, as suggested by Hu et al. ${ }^{6}$ Educational roles should be added to the role profiles of programme coordinators. Role models/ supervisors/mentors play central roles in education, professional development, and PIF of residents by facilitating clinical experiences, teaching formally and informally, and improving the training environment ${ }^{10}$; however, it is sometimes difficult for the supervisors to handle all aspects of the socialization context that may influence resident professional development. Programme coordinators play a supplemental but indispensable role that fosters residents' professional development and identity formation.

The seven key roles of coordinators identified in this study (Table 2) may be related to coordinators' approaches and attributes, and in particular, four attributes may overarch these seven roles (Table 3).

First, a non-hierarchical relationship with residents appears to be the most essential attribute of coordinators. Cruess et al. ${ }^{10}$ described that the most influential factors in facilitating PIF are role models/mentors and the clinical/non-clinical experiences of individual residents; however, the supervisorresident relationship is sometimes a hierarchical one, which may cause problems in the PIF of residents. Our study delineated such cases. We previously reported that when there were conflicts between biomedically-oriented supervisors and psychosocially-oriented residents, this could also discourage residents' PIF. ${ }^{20}$ According to Hofstede, who identified and validated six dimensions of culture worldwide, Japan is a society with a relatively high-power distance, ${ }^{21}$ so a horizontal relationship between the clinical supervisors/mentors and the residents/mentees may be difficult sometimes. ${ }^{22}$ In such 
professional and cultural contexts, the coordinators can intervene and request supervisors to reconsider their teaching methods and their interactions with residents. However, when supervisors or residents themselves show a hierarchical attitude toward the coordinators due to professionally biased attitudes, ${ }^{23}$ it could be difficult to intervene.

Second, parenting attitudes, as described by Hu et al, ${ }^{6}$ would also be a crucial attribute for the coordinators. In the present study, many residents with mental health issues, deficient social skills, and isolation from family and peers were reported, and coordinators presented parenting behaviours such as supporting, fostering, and protecting them. We previously reported that "defending (protecting) residents" was one of the important attributes of clinical teachers in Japan. ${ }^{24}$ Coordinators in this study took the role of protecting residents, as shown in the results (case 5 in the supplementary table: protecting residents from the negative influence of clinical experiences). Furthermore, residents have to establish a new relationship with peers once they graduate from medical schools and start working in different teaching hospitals. However, these relationships are sometimes not easily established, and residents may fear isolation and be sensitive to how they are perceived and treated by their new peers. Coordinators in this study supported such residents (cases 28 to 33 in the supplementary table: providing support for isolated residents). The problems of residents and supporting behaviours of coordinators and supervisors in Japan may be explained by the cultural characteristics of collectivism in Japan, where an emphasis on familial or fellow ties exists. ${ }^{21}$

Third, sensitivity to early and subtle changes in residents' behaviours could help to identify the need for early support and intervention. Many coordinators in this study noticed changes in the appearance of residents, not only in the case of mental health problems, but also in cases of a problematic relationship with supervisors, a decreased self-confidence and dissatisfaction with the programme. Supervisors are usually busy with their clinical responsibility, and the clinical rotations of residents are often short. Thus, changes in the appearance of residents may be overlooked by the clinical staff. Coordinators are able to observe residents over the entire period of training, so they can be sensitive to the residents' facial expressions and mood, and can offer a place with a relaxed atmosphere for the residents to communicate openly and comfortably. Mental health problems are a global issue for health professionals, and a correlation between long working hours and the incidence of depression has been reported. ${ }^{25}$ Residents may not be aware of their own maladaptive responses to stress, and the surrounding staff is more likely to recognize resident stress. ${ }^{26}$ In this sense, coordinators play an important role in detecting early signs of mental stress and fostering a more productive learning environment to mitigate and possibly prevent stressors from leading to more severe mental health problems.

Finally, coordinators are able to observe and assess residents from the perspective of a member of the public. Although coordinators are now regarded as important members of the team training health professionals, they seem to be able to assess resident behaviours more sensitively and efficiently than medical staff, through the lens of society and societal norms. Coordinators could notice residents' 
insufficient social skills or inadequate behaviours as professionals, as Nikels et al. reported, ${ }^{7}$ to lead communication and remediation (cases 21 to 27 in the supplementary table). Some of these behaviours could be learned as residents develop through the residency programme; however, some unprofessional behaviours are derived from their prior personal experiences and awkward identities, and these behaviours are difficult to remediate.

Thus, this study suggests a wide range of educational roles for programme coordinators. Many of the proposed roles are not formally described in the previous reports on the coordinator's role profiles. ${ }^{1,2,3}$ Flynn et al. proposed the establishment of a development programme for staff who support health professional students, especially in newly identified roles such as pastoral and educational roles. ${ }^{27}$ Our study also provides additional evidence of educational roles of programme coordinators from a stand point of PIF and suggests exemplary attributes of coordinators including ability for form non-hierarchical relationships, parenting attitude, sensitivity to changes in residents, and seeing residents from the perspective of a member of the public (Table 3). This will facilitate a revision of the formal role profiles of programme coordinators.

\section{Limitation}

We adopted a semi-structured and web-based anonymous questionnaire to collect cases of educational support from programme coordinators, with consideration for the confidentiality and sensitivity of the cases; however, further studies should directly interview coordinators to confirm and deepen the findings of this study. There could be issues of selection in which cases were reported based on the sensitive nature of some of the cases, thereby impacting our results. Clinical supervisors' and trainees' perception of the roles of coordinators should also be investigated for the triangulation of the results obtained in this study. We investigated resident cases in Japan, a country where cultural characteristics of collectivism and relatively high-power distance may affect the results, this could be a limitation of our study pertaining to its generalizability. Similar studies on the educational support provided by programme coordinators should be conducted in other cultural spheres.

\section{Conclusions}

This study highlights the educational roles of programme coordinators in the professional identity development of residents. Coordinators play important educational roles by communicating with supervisors, protecting/facilitating/supporting residents, and fostering/creating a productive and safe learning environment. Attributes including non-hierarchical relationships, parenting attitudes, sensitivity to changes in residents, and a perspective of a member of the public underpin the educational roles of the coordinators. Our results provide evidence for revising role profiles of programme coordinators and providing targeted professional development training for coordinators.

\section{Abbreviations}


PIF

professional identity formation

\section{Declarations}

\section{Ethics approval and consent to participate}

This study was approved by the Institutional Review Board of Gifu University (approval No. 27-340). The principles of the World Medical Association and the Declaration of Helsinki were applied in this study. Written informed consent was obtained from all participants in this study. All participants were informed that their comments would be kept anonymous and that some of their direct quotations may be included in the paper.

\section{Consent for publication}

Not applicable

\section{Availability of data and materials}

The datasets used and/or analysed for this study are available with the corresponding author upon reasonable request.

\section{Competing interests}

The authors declare that there are no competing interests.

\section{Funding}

There is no funding source to report for this research.

\section{Authors' contributions}

MA and YS contributed to the conception, design, acquisition of data, analysis, interpretation of data, and drafting of the manuscript. HO, CK, RI, and TS made substantial contributions to the design of the study, data analysis and interpretation, and drafting the manuscript critically for important intellectual content. $M A B$ contributed to the design, analysis, and drafting of the manuscript from an international perspective. All authors approved the final manuscript and agreed to be accountable for all aspects of the work.

\section{Acknowledgements}

The authors express their deep gratitude to the Japanese administrators who participated in this study. We also thank Dr Sakae Iwasaki, Japan Council for Evaluation of Postgraduate Clinical Training, for his support, encouragement, and helpful advice. 


\section{References}

1. O'Sullivan PS, Heard JK, Petty M, Cynthia C. Mercado CC, Hicks E. Educational development program for residency program directors and coordinators. Teach Learn Med. 2006;18(2):142-149; doi: 10.1207/ s15328015tIm1802_9.

2. Nickel BL, Roof J, Dolejs S, Choi JN, Torbeck L. Identifying managerial roles of general surgery coordinators: making the case for utilization of a standardized job description framework. J Surg Educ. 2018;75:e38-e46; doi: 10.1016/j.jsurg.2018.07.003.

3. Dubois L, Marsh T, Demers LB. Program coordinator professional development: definition, perception of importance, motivating factors, and barriers. Am J Med. 2019;132:114-118; doi: 10.1016/j.amjmed.2018.09.001.

4. Otterstad D. The role of the residency coordinator. Acad Radiol. 2003;10(suppl 1): S48-S53; doi: 10.1016/s1076-6332(03)80150-2.

5. Grant RE, Murphy LA, Murphy JE. Expansion of the coordinator role in orthopaedic residency program management. Clin Orthop Relat Res. 2008;466:737-742; doi: 10.1007/s11999-007-0110-6.

6. Hu WCY, Flynn E, Mann R, Woodward-Kron R: From paperwork to parenting: experiences of professional in support. Med Educ. 2017;51:290-301; doi: 10.1111/medu.13143.

7. Nikels SM, Guiton G, Loeb D, Brandenburg S: Evaluating non-physician staff members' self-perceived ability to provide multisource evaluation of residents. J Grad Med Educ. 2013;5:64-69; doi: 10.4300/JGME-D-11-00315.1.

8. Aono $\mathrm{M}$, Obara $\mathrm{H}$, Kawakami $\mathrm{C}$, Suzuki Y. Perception of residency program coordinators on their roles and attributes. Med Educ (Japan). 2019;50(6):569-572. (in Japanese)

9. Cruess RL, Cruess SR, Boudreau JD, Snell L, Steinert Y. Reframing medical education to support professional identity formation. Acad Med. 2014;89:1446-1451; doi:

10.1097/ACM.0000000000000427.

10. Cruess RL, Cruess SR, Boudreau JD, Snell L, Steinert Y: A schematic representation of the professional identity formation and socialization of medical student and residents: a guide for medical educators. Acad Med. 2015;90:718-725; doi: 10.1097/ACM.0000000000000700.

11. Suzuki Y, Gibbs T, Fujisaki K. Medical education in Japan: a challenge to the healthcare system: Med Teach. 2008;30(9-10):846-850; doi: 10.1080/01421590802298207.

12. Teo A. The current state of medical education in Japan: a system under reform. Med Educ. 2007;41:302-308; doi: 10.1111/j.1365-2929.2007.02691.x.

13. Nomura K, Yano E, Aoki M, Kawaminami K, Endo H, Fukui T. Improvement of residents' clinical competency after the introduction of new postgraduate medical education program in Japan. Med Teach. 2008;30(6):e161-169; doi: 10.1080/01421590802047307.

14. Braun V, Clarke V. Using thematic analysis in psychology. Qual Res Psychol. 2006;3:77-101; doi: 10.1191/1478088706qp063oa. 
15. Braun V, Clarke V. Reflecting on reflexive thematic analysis. Qual Res Sport Exerc Health. 2019;11:589-597; doi: 10.1080/2159676X.2019.1628806.

16. Mayring P. Qualitative content analysis. Forum Qual Soc Res. 2000;1:20; http://www.qualitativeresearch.net/index.php/fqs/article/view/1089/2385\#gref. Accessed November 6, 2021.

17. Cruess RL, Cruess SR. Professionalism and professional identity formation: the cognitive base. In Cruess RL, Cruess SR, Steinert Y eds. Teaching Medical Professionalism. Cambridge: Cambridge University Press; 2016:5-25.

18. Yao DC, Wright SM. National survey of internal medicine program directors regarding problem residents. JAMA 2000;284:1099-1104.

19. Reamy BV, Harmann JH. Residents in trouble: an in-depth assessment of the 25-year experience of a single family medicine residency. Fam Med 2006;38:252-257.

20. Arai K, Saiki T, Imafuku R, Kawakami C, Fujisaki K, Suzuki Y. What do Japanese residents learn from treating dying patients? The implications for training in end-of-life care. BMC Med Educ. 2017;17:205; doi: 10.1186/s12909-017-1029-6.

21. Hofstede G, Hofstede GJ, Minkov M. Cultures and organizations: software of the mind. 3rd ed. New York, NY: McGraw-Hill; 2010.

22. Obara H, Saiki T, Imafuku R, Fujisaki K, Suzuki Y. Influence of national culture on mentoring relationship: a qualitative study of Japanese physician-scientists. BMC Med Educ. 2021;21:300; doi: 10.1186/s12909-021-02744-2.

23. Papadakis MA, Teherani A, Banach MA, Knettler TR, Rattner SL, Stern DT, et al. Disciplinary action by medical boards and prior behavior in medical school. N Engl J Med. 2005;353:2673-2682; doi: 10.1056/NEJMsa052596.

24. Nishiya K, Sekiguchi S, Yoshimura H, Takamura A, Wada H, Konishi E, et al. Good clinical teachers in pediatrics: the perspective of pediatricians in Japan. Pediatr Intl. 2020;62:549-555; doi: 10.1111/ped.14095.

25. Ogawa R, Seo E, Maeno T, Ito M, Sanuki M, Maeno T. The relationship between long working hours and depression among first-year residents in Japan. BMC Med Educ. 2018;18:50; doi: 10.1186/s12909-018-1171-9.

26. Riesenberg LA, Berg K, Berg D, Morgan CJ, Davis J, Davis R, et al. Resident and attending physician perception of maladaptive response to stress in residents. Med Educ Online. 2014;19:25041; doi: 10.3402/meo.v19.25041.

27. Flynn E, Woodward-Kron R, Hu W. Training for staff who support students. Clin Teach 2016;13:6368; doi: $10.1111 /$ tct.12392.

\section{Supplementary Files}

This is a list of supplementary files associated with this preprint. Click to download. 
- BMC20211106SupplementaryfileYS.docx 